\title{
Computational Procedure of Performance Assessment of Lifetime Index of Products for the Weibull Distribution with the Progressive First-Failure-Censored Sampling Plan
}

\author{
Ching-Wen Hong, ${ }^{1}$ Wen-Chuan Lee, ${ }^{2}$ and Jong-Wuu $\mathrm{Wu}^{3}$ \\ ${ }^{1}$ Department of Information Management, Shih Chien University, Kaohsiung Campus, \\ Kaohsiung 84550, Taiwan \\ ${ }^{2}$ Department of International Business, Chang Jung Christian University, Tainan 71101, Taiwan \\ ${ }^{3}$ Department of Applied Mathematics, National Chiayi University, Chiayi City 60004, Taiwan \\ Correspondence should be addressed to Jong-Wuu Wu, jwwu@mail.ncyu.edu.tw
}

Received 31 January 2012; Accepted 6 March 2012

Academic Editor: Vu Phat

Copyright (C) 2012 Ching-Wen Hong et al. This is an open access article distributed under the Creative Commons Attribution License, which permits unrestricted use, distribution, and reproduction in any medium, provided the original work is properly cited.

Process capability analysis has been widely applied in the field of quality control to monitor the performance of industrial processes. In practice, lifetime performance index $C_{L}$ is a popular means to assess the performance and potential of their processes, where $L$ is the lower specification limit. This study will apply the large-sample theory to construct a maximum likelihood estimator (MLE) of $C_{L}$ with the progressive first-failure-censored sampling plan under the Weibull distribution. The MLE of $C_{L}$ is then utilized to develop a new hypothesis testing procedure in the condition of known $L$.

\section{Introduction}

Effectively managing and measuring the business operational process is widely seen as a means of ensuring business survival through reduced time to market, increased quality, and reduced costs. Process capability analysis is an effective means of measuring process performance and potential capability. In the manufacturing industry, process capability indices are utilized to assess whether product quality meets the required level. For instance, Montgomery [1] (or Kane [2]) proposed the process capability index $C_{L}$ (or $C_{P L}$ ) for evaluating the lifetime performance of electronic components, where $L$ is the lower specification limit, since the lifetime of electronic components exhibits the largerthe-better quality characteristic of time orientation. Tong et al. [3] constructed a uniformly 
minimum variance unbiased estimator (UMVUE) of $C_{L}$ under an exponential distribution. Moreover, the UMVUE of $C_{L}$ is then utilized to develop the hypothesis testing procedure. The purchasers can then employ the testing procedure to determine whether the lifetime of electronic components adheres to the required level. Manufacturers can also utilize this procedure to enhance process capability. Hong et al. [4] also constructed a maximum likelihood estimator (MLE) of $C_{L}$ with the type II right censored sample under a pareto distribution. Moreover, the MLE estimator of $C_{L}$ is then utilized to develop a hypothesis testing procedure. The managers can then employ the testing procedure to assess the business performance. Lee et al. $[5,6]$ also constructed an MLE of $C_{L}$ under the Burr XII distribution with progressively type II right censored sample and the Gompertz distribution with the firstfailure-censored sample, respectively. Moreover, the MLE of $C_{L}$ is then utilized to develop a hypothesis testing procedure. The managers can then employ the testing procedure to assess the quality performance of product.

In this study, process capability analysis is also utilized to assess product quality. The lifetime performance index $C_{L}$ is also utilized to measure product quality with the Weibull distribution based on the progressive first-failure-censored sampling plan. The Weibull distribution is useful in a great variety of applications, particularly as a model for product life. It has also been used as the distribution of strength of certain materials. It is named after Weibull [7], who popularized its use among engineers. One reason for its popularity is that it has a great variety of shapes. This makes it extremely flexible in fitting data, and it empirically fits many kinds of data (see Nelson [8]). The Weibull distribution includes the exponential and the Rayleigh distributions as special cases. The exponential and the Rayleigh distributions have been recognized as a useful model for the analysis of lifetime data. The Weibull distribution family has played an important role in the analysis of lifetime data. The probability density function (p.d.f.) and the cumulative distribution function (c.d.f.) of the Weibull distribution are as follows, respectively,

$$
\begin{gathered}
f_{X}(x)=\frac{\beta}{\alpha^{\beta}} x^{\beta-1} \exp \left[-\left(\frac{x}{\alpha}\right)^{\beta}\right], \quad x>0, \alpha>0, \beta>0, \\
F_{X}(x)=1-\exp \left[-\left(\frac{x}{\alpha}\right)^{\beta}\right], \quad x>0, \alpha>0, \beta>0 .
\end{gathered}
$$

The parameter $\beta$ is called the shape parameter, and the parameter $\alpha$ is called the scale parameter. For the special case $\beta=1$, the Weibull distribution is the simple exponential distribution. For the special case $\beta=2$, the Weibull distribution is the Rayleigh distribution. In addition, for $3 \leq \beta \leq 4$, the shape of the Weibull distribution is close to that of the normal distribution (see Nelson [8]).

In life testing experiments, the experimenter may not always be in a position to observe the life times of all the products (or items) put on test. This may be because of time limitation and/or other restrictions (such as money, material resources, mechanical or experimental difficulties) on data collection. Therefore, censored samples may arise in practice. In this study, we consider the case of progressive first-failure-censored sampling plan. The progressive first-failure-censored sampling plan is the combination of first-failurecensored sampling plan and progressively type II right censored sampling plan. Owing to, sometimes the lifetime of a product is quite long. Thus, a right type II censored sample plan for such a product can be too long. Johnson [9] proposed the first-failure-censored sampling plan in which the experimenter can decide to group the test units into several sets (each set 
is an assembly of test units), and then run all the test units simultaneously until the first failure in each group. Such plans are usually feasible when test facilities are scarce but test material is relatively cheap. Balasooriya [10] examined the failure-censored sampling plan for the 2-parameter exponential distribution based on testing $r$ random samples, each of size $n$, one after the other. That procedure is based on exact results, and only the first failure time of each sample is needed. The Balasooriya sampling plan is compared with traditional sampling plans using a sample of size $r \cdot n$ (see Wu et al. [11]). The first-failure-censored sampling plan has an advantage in terms of shorter test time and a saving of resources. Note that a first-failure-censoring scheme is terminated when the first failure in each set is observed. If an experimenter desires to remove some sets of test units before observing the first failures in these sets, the above-described scheme will not be of use to the experimenter. The firstfailure-censored sampling plan does not allow for sets to be removed from the test at the points other than the final termination point. However, this allowance will be desirable when some sets of the surviving units in the experiment that are removed early on can be used for some other tests. As in the case of accidental breakage of experimental units or loss of contact with individuals under study, the loss of test units at points other than the termination point may also be unavoidable (see Wu and Kuss [12]). Therefore, we also consider the case of the progressively type II right censoring in this study. Progressive type II right censoring is a useful scheme in which a specific fraction of individuals at risk may be removed from the experiment at each of several ordered failure times (see Fernández [13]). The experimenter can remove units from a life test at various stages during the experiments, possibly resulting in a saving of costs and time (see Sen [14]). Therefore, the progressive first-failure-censored sampling plan has an advantage in terms of shorter test time, a saving of resources, and in which a specific fraction of individuals at risk may be removed from the experiment at each of several ordered failure times. The progressive first-failure-censored sampling plan is illustrated as follows.

Suppose that $m$ is the number of failures observed before termination and $n$ independent groups with $k$ items within each group are put in a life test. $R_{1}$ groups and the group in which the first failure is observed are randomly removed from the test as soon as the first failure (say $X_{1}$ ) has occurred, $R_{2}$ groups and the group in which the second failure is observed are randomly removed from the test as soon as the first failure (say $X_{2}$ ) has occurred, and finally $R_{m}(m \leq n)$ groups and the group in which the $m$ th failure is observed are randomly removed from the test as soon as the $m$ th failure (say $X_{m}$ ) has occurred. Then $X_{1} \leq X_{2} \leq \cdots \leq X_{m}$ are called the progressive first-failure-censored order statistics with censoring scheme $R=\left(R_{1}, R_{2}, \ldots, R_{m}\right)$. It is clear that $n=m+R_{1}+R_{2}+\cdots+R_{m}$. The familiar complete, type II right censored, first-failure-censored, and progressively type II right censored samples are special cases of the progressive first-failure-censored sampling plan. Note that if $R_{1}=R_{2}=\cdots=R_{m}=0$, then the progressive first-failure-censored sampling plan reduces to the first-failure-censored sampling plan. If $k=1$, then the progressive first-failurecensored sampling plan reduces to the progressively type II right censored sampling plan. If $k=1$ and $R_{1}=R_{2}=\cdots=R_{m}=0$, then $n=m$ and the progressive first-failure-censored sampling plan reduces to the complete sampling plan. If $k=1, R_{1}=R_{2}=\cdots=R_{m-1}=0$, and $R_{m}=n-m$, then the progressive first-failure-censored sampling plan reduces to type II right censored sampling plan (see Wu and Kuş [12]).

Hong et al. [4], and Lee et al. [5, 6] proposed the data transformation method to construct a MLE of $C_{L}$. In this study, the large sample in place of the data transformation method. Under the assumption of Weibull distribution, the main aim of this paper will apply the large-sample theory to construct an MLE of $C_{L}$ with the progressive first-failure-censored 
sampling plan. The MLE of $C_{L}$ is then utilized to develop a new hypothesis testing procedure in the condition of known $L$. The new testing procedure can be employed by managers to assess whether the lifetime of products adheres to the required level in the condition of known $L$.

The rest of this paper is organized as follows. Section 2 introduces some properties of the lifetime performance index for lifetime of product with the Weibull distribution. Section 3 discusses the relationship between the lifetime performance index and conforming rate. Section 4 then presents the MLE of the lifetime performance index and its statistical properties with Weibull distribution based on the progressive first-failure-censored sampling plan. Section 5 will apply the large-sample theory to develop a new hypothesis testing procedure for the lifetime performance index. One numerical example and concluding remarks are made in Sections 6 and 7, respectively.

\section{The Lifetime Performance Index}

Suppose that the lifetime (in years) of products may be modeled by a Weibull distribution. Let $X$ denote the lifetime of such a product and $X$ has the Weibull distribution with the p.d.f. as given in (1.1). Clearly, a longer lifetime implies a better product quality. Hence, the lifetime is a larger-the-better-type quality characteristic. The lifetime is generally required to exceed $L$ unit times to both be economically profitable and satisfy customers. Montgomery [1] developed a capability index $C_{L}$ for properly measuring the larger-the-better quality characteristic. $C_{L}$ is defined as follows:

$$
C_{L}=\frac{\mu-L}{\sigma}
$$

where the process mean is $\mu$, the process standard deviation is $\sigma$, and $L$ is the lower specification limit.

To assess the lifetime performance of products, $C_{L}$ can be defined as the lifetime performance index. Under $X$ has the Weibull distribution and there are several important properties, as follows.

(i) The lifetime performance index $C_{L}$ can be rewritten as

$$
\begin{gathered}
C_{L}=\frac{\mu-L}{\sigma}=\frac{\alpha \Gamma(1 / \beta+1)-L}{\sqrt{\alpha^{2} \Gamma(2 / \beta+1)-\alpha^{2} \Gamma^{2}(1 / \beta+1)}}, \\
C_{L}<\frac{\Gamma(1 / \beta+1)}{\sqrt{\Gamma(2 / \beta+1)-\Gamma^{2}(1 / \beta+1)}},
\end{gathered}
$$

where the process mean $\mu=E(X)=\alpha \Gamma(1 / \beta+1)$, the process standard deviation $\sigma=\sqrt{\operatorname{Var}(X)}=\sqrt{\alpha^{2} \Gamma(2 / \beta+1)-\alpha^{2} \Gamma^{2}(1 / \beta+1)}, L$ is the lower specification limit, and the gamma function $\Gamma(\lambda)=\int_{0}^{\infty} y^{\lambda-1} e^{-y} d y$ for $\lambda>0$. 
(ii) The failure rate function $r_{X}(x)$ is defined by

$$
r_{X}(x)=\frac{f_{X}(x)}{1-F_{X}(x)}=\frac{\left(\beta / \alpha^{\beta}\right) x^{\beta-1} \exp \left[-(x / \alpha)^{\beta}\right]}{\exp \left[-(x / \alpha)^{\beta}\right]}=\frac{\beta}{\alpha^{\beta}} x^{\beta-1}, \quad x>0, \alpha>0, \beta>0 .
$$

When the mean $\alpha \Gamma(1 / \beta+1)(>L)$, then the lifetime performance index $C_{L}>0$. From (2.2) and (2.3), we can see that, for example, as given $\beta>0$, the larger $\alpha$ (i.e., the larger the mean $\alpha \Gamma(1 / \beta+1)$ ), then the smaller the failure rate and the lager the lifetime performance index $C_{L}$. Therefore, the lifetime performance index $C_{L}$ reasonably and accurately represents the lifetime performance of new product.

\section{The Conforming Rate}

If the lifetime of a product $X$ exceeds the lower specification limit $L$, then the product is defined as a conforming product. The ratio of conforming products is known as the conforming rate and can be defined as

$$
\begin{aligned}
P_{r} & =P(X>L)=\int_{L}^{\infty} \frac{\beta}{\alpha^{\beta}} x^{\beta-1} \exp \left[-\left(\frac{x}{\alpha}\right)^{\beta}\right] d x \\
& =\exp \left\{-\left[\Gamma\left(\frac{1}{\beta}+1\right)-C_{L} \sqrt{\Gamma\left(\frac{2}{\beta}+1\right)-\Gamma^{2}\left(\frac{1}{\beta}+1\right)}\right]^{\beta}\right\},
\end{aligned}
$$

where $C_{L}<\Gamma(1 / \beta+1) / \sqrt{\Gamma(2 / \beta+1)-\Gamma^{2}(1 / \beta+1)}$ and $\beta>0$.

Obviously, a strictly increasing relationship exists between conforming rate $P_{r}$ and the lifetime performance index $C_{L}$ with given $\beta$. Since a one-to-one mathematical relationship exists between the conforming rate $P_{r}$ and the lifetime performance index $C_{L}$, therefore, utilizing the one-to-one relationship between $P_{r}$ and $C_{L}$, lifetime performance index can be a flexible and effective tool, not only for evaluating product quality, but also for estimating the conforming rate $P_{r}$. For given $\beta$ and $C_{L}$, the conforming rate $P_{r}$ can calculated by (3.1).

\section{Maximum Likelihood Estimator of Lifetime Performance Index}

In lifetime testing experiments of products, the experimenter may not always be in a position to observe the lifetimes of all the items on test due to time limitation and/or other restrictions (such as money, material resources, mechanical or experimental difficulties) on data collection. In this study, we consider the case of the progressive first-failure-censored sampling plan. The progressive first-failure-censored sampling plan has an advantage in terms of shorter test time, a saving of resources, and in which a specific fraction of individuals at risk may be removed from the experiment at each of several ordered failure times. 
Let $X$ denote the lifetime of such a product, and $X$ has a Weibull distribution with the p.d.f. $f_{X}(x)$ as (1.1) and c.d.f. $F_{X}(x)$ as (1.2). $X_{1}, X_{2}, \ldots, X_{m}$ are the progressively firstfailure-censored order statistics from the Weibull distribution with censoring scheme $R=$ $\left(R_{1}, R_{2}, \ldots, R_{m}\right)$. Since the joint p.d.f. of $X_{1}, X_{2}, \ldots, X_{m}$ is given as follows:

$$
\begin{aligned}
& f\left(X_{1}, X_{2}, \ldots, X_{m}\right) \\
& \quad=c k^{m} \prod_{j=1}^{m} f\left(X_{j}\right)\left[1-F\left(X_{j}\right)\right]^{k\left(R_{j}+1\right)-1} \\
& \quad=c k^{m} \prod_{j=1}^{m}\left\{\frac{\beta}{\alpha^{\beta}} X_{j}^{\beta-1}\left\{\exp \left[-\left(\frac{X_{j}}{\alpha}\right)^{\beta}\right]\right\}^{k\left(R_{j}+1\right)}\right\}, \quad 0<X_{1} \leq X_{2} \leq \cdots \leq X_{m}<\infty,
\end{aligned}
$$

where $c=n\left(n-R_{1}-1\right)\left(n-R_{1}-R_{2}-2\right) \cdots\left(n-R_{1}-R_{2}-\cdots-R_{m-1}-m+1\right)$, so, the likelihood function is

$$
L(\alpha, \beta)=c k^{m} \prod_{j=1}^{m}\left\{\frac{\beta}{\alpha^{\beta}} X_{j}^{\beta-1}\left\{\exp \left[-\left(\frac{X_{j}}{\alpha}\right)^{\beta}\right]\right\}^{k\left(R_{j}+1\right)}\right\}
$$

The log-likelihood function is

$$
\ln L(\alpha, \beta)=\ln c k^{m}+m \ln \beta-m \beta \ln \alpha+(\beta-1) \sum_{j=1}^{m} \ln X_{j}-k \sum_{j=1}^{m}\left(R_{j}+1\right)\left(\frac{X_{j}}{\alpha}\right)^{\beta}
$$

Assuming that $\alpha$ and $\beta$ are both unknown, the differentiation of (4.3) with respect to $\alpha$ and $\beta$ yields

$$
\begin{gathered}
\frac{\partial \ln L(\alpha, \beta)}{\partial \alpha}=-\frac{m \beta}{\alpha}+k \sum_{j=1}^{m}\left(R_{j}+1\right) X_{j}^{\beta} \beta \alpha^{-\beta-1}, \\
\frac{\partial \ln L(\alpha, \beta)}{\partial \beta}=\frac{m}{\beta}-m \ln \alpha+\sum_{j=1}^{m} \ln X_{j}-k \sum_{j=1}^{m}\left(R_{j}+1\right)\left(\frac{X_{j}}{\alpha}\right)^{\beta} \ln \left(\frac{X_{j}}{\alpha}\right) .
\end{gathered}
$$

The maximum likelihood estimator (MLE) $\widehat{\alpha}$ of $\alpha$ and the MLE $\widehat{\beta}$ of $\beta$ can be derived by solving the equations

$$
\begin{gathered}
-\frac{m \widehat{\beta}}{\widehat{\alpha}}+k \sum_{j=1}^{m}\left(R_{j}+1\right) X_{j}^{\widehat{\beta}} \widehat{\beta} \widehat{\alpha}^{-\widehat{\beta}-1}=0 \\
\frac{m}{\widehat{\beta}}-m \ln \widehat{\alpha}+\sum_{j=1}^{m} \ln X_{j}-k \sum_{j=1}^{m}\left(R_{j}+1\right)\left(\frac{X_{j}}{\widehat{\alpha}}\right)^{\widehat{\beta}} \ln \left(\frac{X_{j}}{\widehat{\alpha}}\right)=0 .
\end{gathered}
$$


By (4.5), the MLE $\widehat{\alpha}$ of $\alpha$ is given by

$$
\widehat{\alpha}=\left[\frac{k \sum_{j=1}^{m}\left(R_{j}+1\right) X_{j}^{\hat{\beta}}}{m}\right]^{1 / \widehat{\beta}} .
$$

The substitution of (4.7) into (4.6) yields the equation

$$
\frac{1}{\widehat{\beta}}+\frac{1}{m} \sum_{j=1}^{m} \ln X_{j}-\frac{\sum_{j=1}^{m}\left(R_{j}+1\right) X_{j}^{\hat{\beta}} \ln X_{j}}{\sum_{j=1}^{m}\left(R_{j}+1\right) X_{j}^{\widehat{\beta}}}=0 .
$$

By (4.8), the MLE $\hat{\beta}$ of $\beta$ can be found by Newton's method.

By using the invariance of MLE (see Zehna [15]), the MLE of $C_{L}$ can be written as

$$
\widehat{C}_{L}=\frac{\widehat{\alpha} \Gamma(1 / \widehat{\beta}+1)-L}{\sqrt{\widehat{\alpha}^{2} \Gamma(2 / \widehat{\beta}+1)-\widehat{\alpha}^{2} \Gamma^{2}(1 / \widehat{\beta}+1)}}, \quad \widehat{C}_{L}<\frac{\Gamma(1 / \widehat{\beta}+1)}{\sqrt{\Gamma(2 / \widehat{\beta}+1)-\Gamma^{2}(1 / \widehat{\beta}+1)}},
$$

where the MLEs $\widehat{\alpha}$ and $\widehat{\beta}$ can be found by Newton's method with (4.7) and (4.8).

The asymptotic normal distribution for the $\widehat{C}_{L}$ can be obtained in large-sample theory. From the log-likelihood function in (4.3), we have

$$
\begin{gathered}
\frac{\partial^{2} \ln L(\alpha, \beta)}{\partial \alpha^{2}}=\frac{m \beta}{\alpha^{2}}-\frac{k \beta(\beta+1)}{\alpha^{2}} \sum_{j=1}^{m}\left(R_{j}+1\right)\left(\frac{X_{j}}{\alpha}\right)^{\beta}, \\
\frac{\partial^{2} \ln L(\alpha, \beta)}{\partial \alpha \partial \beta}=\frac{-m}{\alpha}+\frac{k}{\alpha} \sum_{j=1}^{m}\left(R_{j}+1\right)\left(\frac{X_{j}}{\alpha}\right)^{\beta}\left[\beta \ln \left(\frac{X_{j}}{\alpha}\right)+1\right], \\
\frac{\partial^{2} \ln L(\alpha, \beta)}{\partial \beta^{2}}=\frac{-m}{\beta^{2}}-k \sum_{j=1}^{m}\left(R_{j}+1\right)\left(\frac{X_{j}}{\alpha}\right)^{\beta}\left[\ln \left(\frac{X_{j}}{\alpha}\right)\right]^{2} .
\end{gathered}
$$

And the Fisher information matrix is given by

$$
I(\alpha, \beta)=\left[\begin{array}{ll}
-E\left(\frac{\partial^{2} \ln L(\alpha, \beta)}{\partial \alpha^{2}}\right) & -E\left(\frac{\partial^{2} \ln L(\alpha, \beta)}{\partial \alpha}\right) \\
-E\left(\frac{\partial^{2} \ln L(\alpha, \beta)}{\partial \alpha \partial \beta}\right) & -E\left(\frac{\partial^{2} \ln L(\alpha, \beta)}{\partial \beta^{2}}\right)
\end{array}\right] .
$$

Under some mild regularity conditions (see Theorem 5.2.2 of Sen and Singer [16]), $(\widehat{\alpha}, \widehat{\beta})$ is asymptotically bivariately normal distribution with mean $(\alpha, \beta)$ and covariance matrix $I^{-1}(\alpha, \beta)$, that is, $(\widehat{\alpha}, \widehat{\beta}) \stackrel{D}{\rightarrow} N\left((\alpha, \beta), I^{-1}(\alpha, \beta)\right)$. 
Let

$$
C_{L}=\frac{\alpha \Gamma(1 / \beta+1)-L}{\sqrt{\alpha^{2} \Gamma(2 / \beta+1)-\alpha^{2} \Gamma^{2}(1 / \beta+1)}} \equiv h(\alpha, \beta)
$$

By using the delta method (see Casella and Berger [17, page 245, Theorem 5.5.28]), we have

$$
\widehat{C}_{L}-C_{L} \stackrel{D}{\rightarrow} N\left(0,\left[\frac{\partial h(\alpha, \beta)}{\partial \alpha}, \frac{\partial h(\alpha, \beta)}{\partial \beta}\right] I^{-1}(\alpha, \beta)\left[\frac{\partial h(\alpha, \beta)}{\partial \alpha}, \frac{\partial h(\alpha, \beta)}{\partial \beta}\right]^{T}\right)
$$

And by using Theorem 5.6.1 of Sen and Singer [16] (or Lawless [18, page 549]),

$$
\frac{\left(\widehat{C}_{L}-C_{L}\right)^{2}}{\operatorname{Var}(h(\widehat{\alpha}, \widehat{\beta}))} \stackrel{D}{\longrightarrow} x_{1}^{2}
$$

where

$$
\begin{aligned}
\operatorname{Var}(h(\widehat{\alpha}, \widehat{\beta}))= & {\left[\frac{\partial h(\alpha, \beta)}{\partial \alpha}, \frac{\partial h(\alpha, \beta)}{\partial \beta}\right]_{\substack{\alpha=\widehat{\alpha} \\
\beta=\widehat{\beta}}} I^{-1}(\widehat{\alpha}, \widehat{\beta})\left[\frac{\partial h(\alpha, \beta)}{\partial \alpha}, \frac{\partial h(\alpha, \beta)}{\partial \beta}\right]_{\substack{\alpha=\widehat{\alpha} \\
\beta=\widehat{\beta}}}^{T} } \\
& \frac{\partial h(\alpha, \beta)}{\partial \alpha}=\frac{L}{\alpha^{2} \sqrt{\Gamma(2 / \beta+1)-\Gamma^{2}(1 / \beta+1)}},
\end{aligned}
$$

$$
\begin{aligned}
\frac{\partial h(\alpha, \beta)}{\partial \beta} & \\
= & \frac{-\beta^{-2} \Gamma(1 / \beta+1) \Psi(1 / \beta+1)}{\sqrt{\Gamma(2 / \beta+1)-\Gamma^{2}(1 / \beta+1)}} \\
& -\frac{\beta^{-2}[\alpha \Gamma(1 / \beta+1)-L]\left[-\Gamma(2 / \beta+1) \Psi(2 / \beta+1)+\Gamma^{2}(1 / \beta+1) \Psi(1 / \beta+1)\right]}{\alpha\left[\Gamma(2 / \beta+1)-\Gamma^{2}(1 / \beta+1)\right]^{3 / 2}},
\end{aligned}
$$

the digamma function $\Psi(x)=\Gamma^{\prime}(x) / \Gamma(x), x>0$, and the observed information matrix

$$
I(\widehat{\alpha}, \hat{\beta})=\left[\begin{array}{cc}
-\frac{\partial^{2} \ln L(\alpha, \beta)}{\partial \alpha^{2}} & -\frac{\partial^{2} \ln L(\alpha, \beta)}{\partial \alpha \partial \beta} \\
-\frac{\partial^{2} \ln L(\alpha, \beta)}{\partial \alpha \partial \beta} & -\frac{\partial^{2} \ln L(\alpha, \beta)}{\partial \beta^{2}}
\end{array}\right]_{\substack{\alpha=\widehat{\alpha} \\
\beta=\widehat{\beta}}} .
$$




\section{Testing Procedure for the Lifetime Performance Index}

Construct a statistical testing procedure to assess whether the lifetime performance index adheres to the required level. Assuming that the required index value of lifetime performance is larger than $c$, where $c$ denotes the target value, the null hypothesis $H_{0}: C_{L} \leq c$ and the alternative hypothesis $H_{1}: C_{L}>c$ are constructed.

Firstly, by using $\widehat{C}_{L}$, the MLE of $C_{L}$ as the test statistic, the rejection region can be expressed as $\left\{\widehat{C}_{L}>C_{0}\right\}$. Given the specified significance level $\alpha^{*}$, the critical value $C_{0}$ can be calculated as follows:

$$
\begin{aligned}
& \operatorname{Sup}_{\left\{C_{L} \leq c\right\}} P\left(\widehat{C}_{L}>C_{0}\right) \leq \alpha^{*}, \\
& \Longrightarrow P\left(\frac{\hat{C}_{L}-C_{L}}{\sqrt{\operatorname{Var}(h(\widehat{\alpha}, \hat{\beta}))}}>\frac{C_{0}-C_{L}}{\sqrt{\operatorname{Var}(h(\hat{\alpha}, \widehat{\beta}))}} \mid C_{L} \leq c\right) \leq \alpha^{*}, \\
& \Longrightarrow P\left(\frac{\widehat{C}_{L}-C_{L}}{\sqrt{\operatorname{Var}(h(\hat{\alpha}, \hat{\beta}))}}>\frac{C_{0}-C_{L}}{\sqrt{\operatorname{Var}(h(\widehat{\alpha}, \hat{\beta}))}} \mid C_{L}=c\right)=\alpha^{*}, \\
& \Longrightarrow P\left(\frac{\widehat{C}_{L}-c}{\sqrt{\operatorname{Var}(h(\widehat{\alpha}, \widehat{\beta}))}}>\frac{C_{0}-c}{\sqrt{\operatorname{Var}(h(\widehat{\alpha}, \widehat{\beta}))}}\right)=\alpha^{*}, \\
& \Longrightarrow P\left(\left(\frac{\widehat{C}_{L}-c}{\sqrt{\operatorname{Var}(h(\widehat{\alpha}, \hat{\beta}))}}\right)^{2}>\left(\frac{C_{0}-c}{\sqrt{\operatorname{Var}(h(\widehat{\alpha}, \hat{\beta}))}}\right)^{2}\right)=\alpha^{*}, \\
& \Longrightarrow P\left(\left(\frac{\widehat{C}_{L}-c}{\sqrt{\operatorname{Var}(h(\widehat{\alpha}, \widehat{\beta}))}}\right)^{2} \leq\left(\frac{C_{0}-c}{\sqrt{\operatorname{Var}(h(\widehat{\alpha}, \widehat{\beta}))}}\right)^{2}\right)=1-\alpha^{*},
\end{aligned}
$$

where

$$
\operatorname{Var}(h(\widehat{\alpha}, \widehat{\beta}))=\left[\frac{\partial h(\alpha, \beta)}{\partial \alpha}, \frac{\partial h(\alpha, \beta)}{\partial \beta}\right]_{\substack{\alpha=\alpha \\ \beta=\widehat{\beta}}} I^{-1}(\widehat{\alpha}, \widehat{\beta})\left[\frac{\partial h(\alpha, \beta)}{\partial \alpha}, \frac{\partial h(\alpha, \beta)}{\partial \beta}\right]_{\substack{\alpha=\widehat{\alpha} \\ \beta=\hat{\beta}}}^{T}
$$

and under

$$
C_{L}=c, \quad\left(\frac{\widehat{C}_{L}-c}{\sqrt{\operatorname{Var}(h(\widehat{\alpha}, \hat{\beta}))}}\right)^{2} \stackrel{D}{\rightarrow} x_{(1)}^{2} .
$$


From (5.1), by utilizing CHIINV $\left(1-\alpha^{*}\right)$ function which represents the lower $100\left(1-\alpha^{*}\right)$ th percentile of $x_{(1)}^{2}$, then

$$
\left(\frac{C_{0}-c}{\sqrt{\operatorname{Var}(h(\widehat{\alpha}, \hat{\beta}))}}\right)^{2}=\operatorname{CHIINV}\left(1-\alpha^{*}\right)
$$

is obtained. Thus, the following critical value can be derived:

$$
C_{0}=c+\sqrt{\operatorname{Var}(h(\widehat{\alpha}, \hat{\beta}))} \sqrt{\operatorname{CHIINV}\left(1-\alpha^{*}\right)},
$$

where $c$ and $\alpha^{*}$ denote the target value and the specified significance level, respectively.

The managers can then employ the one-sided hypothesis testing to determine whether the lifetime performance index adheres to the required level. The proposed testing procedure about $C_{L}$ can be organized as follows.

Step 1. Determine the lower lifetime limit $L$ for products and performance index value $c$; then the testing null hypothesis $H_{0}: C_{L} \leq c$ and the alternative hypothesis $H_{1}: C_{L}>c$ are constructed.

Step 2. Specify a significance level $\alpha^{*}$.

Step 3. Calculate the value of test statistic

$$
\widehat{C}_{L}=\frac{\widehat{\alpha} \Gamma(1 / \widehat{\beta}+1)-L}{\sqrt{\widehat{\alpha}^{2} \Gamma(2 / \widehat{\beta}+1)-\widehat{\alpha}^{2} \Gamma^{2}(1 / \widehat{\beta}+1)}}, \quad \widehat{C}_{L}<\frac{\Gamma(1 / \widehat{\beta}+1)}{\sqrt{\Gamma(2 / \widehat{\beta}+1)-\Gamma^{2}(1 / \widehat{\beta}+1)}}
$$

where the MLEs $\widehat{\alpha}$ and $\widehat{\beta}$ can be found by Newton's method with (4.7) and (4.8).

Step 4. Obtain the critical value

$$
C_{0}=c+\sqrt{\operatorname{Var}(h(\widehat{\alpha}, \hat{\beta}))} \sqrt{\operatorname{CHIINV}\left(1-\alpha^{*}\right)},
$$

where

$$
\operatorname{Var}(h(\widehat{\alpha}, \widehat{\beta}))=\left[\frac{\partial h(\alpha, \beta)}{\partial \alpha}, \frac{\partial h(\alpha, \beta)}{\partial \beta}\right]_{\substack{\alpha=\widehat{\alpha} \\ \beta=\widehat{\beta}}} I^{-1}(\widehat{\alpha}, \widehat{\beta})\left[\frac{\partial h(\alpha, \beta)}{\partial \alpha}, \frac{\partial h(\alpha, \beta)}{\partial \beta}\right]_{\substack{\alpha=\widehat{\alpha} \\ \beta=\widehat{\beta}}}^{T}
$$

and $c$ and $\alpha^{*}$ denote the target value and the specified significance level. 
Step 5. The decision rule of statistical test is provided as follows.

If $\widehat{C}_{L}>C_{0}$, it is concluded that the lifetime performance index of product meets the required level.

Based on the proposed testing procedure, the lifetime performance of products is easy to assess. One numerical example of the proposed testing procedure is given in Section 6, and the numerical examples illustrate the use of the testing procedure.

\section{Numerical Examples}

In this section, we propose the new hypothesis testing procedure to one simulated largesample data set. Example 6.1 considered is a simulated large-sample data with $k=5, n=50$, and $m=30$ from a Weibull distribution.

Example 6.1. The following data are the progressive first-failure-censored sample of a computer generated from a Weibull distribution with p.d.f. as given in (1.1) and $\alpha=40, \beta=1$, $L=4, C_{L}=0.9$. The simulated progressive first-failure-censored sample and the simulated progressive first-failure-censored scheme are given as follows:

$\left\{x_{i}, i=1, \ldots, 30\right\}=\{0.10971,0.11117,0.78476,1.27366,1.30471,1.78242,1.85144$, $1.88851,2.70589,2.93703,3.53395,3.65632,3.76333,4.10132,4.50531,4.94733,5.06265,7.04528$, 7.52044, 8.08150, 9.07310, 9.27218, 10.6786, 11.7043, 12.4732, 13.1637, 13.8520, 13.9263, 14.7226, $19.5564\}, R=(0,0,1,0,0,2,0,1,0,0,3,0,0,5,0,1,0,0,3,0,0,1,0,0,0,0,2,0,0,1), k=5$, $n=50$, and $m=30$.

Then, we also state the proposed testing procedure about $C_{L}$ as follows.

Step 1. The lower lifetime limit $L$ is assumed to be 4 by the simulation condition $L=4$, that is, if the lifetime of a product exceeds 4 , then the product is defined as a conforming product. To deal with the product managers' concerns regarding operational performance, the conforming rate $P_{r}$ of operational performances is required to exceed 80 percent. By the simulation condition $\beta=1$ and (3.1), the $C_{L}$ value of the operational performances is required to exceed 0.78 . Thus, the performance index value is set at $c=0.78$. The testing hypothesis $H_{0}: C_{L} \leq 0.78$ versus $H_{1}: C_{L}>0.78$ is constructed.

Step 2. Specify a significance level $\alpha^{*}=0.05$.

Step 3. Calculate the value of test statistic

$$
\begin{aligned}
\widehat{C}_{L} & =\frac{\widehat{\alpha} \Gamma(1 / \widehat{\beta}+1)-L}{\sqrt{\widehat{\alpha}^{2} \Gamma(2 / \widehat{\beta}+1)-\widehat{\alpha}^{2} \Gamma^{2}(1 / \widehat{\beta}+1)}} \\
& =\frac{40.3104 \Gamma(1 / 1.17825+1)-4}{\sqrt{40.3104^{2} \Gamma(2 / 1.17825+1)-40.3104^{2} \Gamma^{2}(1 / 1.17825+1)}} \\
& =1.30537,
\end{aligned}
$$

where the MLEs $\widehat{\alpha}=40.3104$ and $\widehat{\beta}=1.17825$ can be found by Newton's method with (4.7) and (4.8). 
Step 4. Obtain the critical value

$$
\begin{aligned}
C_{0} & =0.78+\sqrt{\operatorname{Var}(h(40.3104,1.17825))} \sqrt{\operatorname{CHIINV}(1-0.05)} \\
& =0.78+\sqrt{0.013501} \times \sqrt{3.841} \\
& =1.00774
\end{aligned}
$$

according to

$$
\begin{aligned}
& I_{0}{ }^{-1}(40.3104,1.17825)=\left[\begin{array}{ll}
0.025631 & 1.29510 \\
1.29510 & 98.9497
\end{array}\right]^{-1}=\left[\begin{array}{cc}
115.206 & -1.50786 \\
-1.50786 & 0.029842
\end{array}\right], \\
& {\left[\frac{\partial h(\alpha, \beta)}{\partial \alpha}, \frac{\partial h(\alpha, \beta)}{\partial \beta}\right]_{\substack{\alpha=40.3104 \\
\hat{\beta}=1.17825}}=[0.003057811,0.81798], } \\
& \operatorname{Var}(h(40.3104,1.17825))=[0.003057811,0.81798]\left[\begin{array}{cc}
115.206 & -1.50786 \\
-1.50786 & 0.029842
\end{array}\right]\left[\begin{array}{c}
0.003057811 \\
0.81798
\end{array}\right] \\
&=0.013501,
\end{aligned}
$$

the target value $c=0.78$, and the significance level $\alpha^{*}=0.05$.

Step 5. Because $\widehat{C}_{L}=1.30537>C_{0}=1.00774$, so we do reject to the null hypothesis $H_{0}: C_{L} \leq$ 0.78 . Thus, we can conclude that the lifetime performance index of product does meet the required level.

\section{Conclusions}

Process capability analysis has been widely applied in the field of quality control to monitor the performance of industrial processes. In practice, lifetime performance index $C_{L}$ is a popular means to assess the performance and potential of their processes, where $L$ is the lower specification limit. Moreover, in life testing experiments, the experimenter may not always be in a position to observe the life times of all the businesses (or items) put on test. This may be because of time limitation and/or other restrictions (such as money, material resources, mechanical or experimental difficulties) on data collection. Therefore, censored samples may arise in practice. The progressive first-failure-censored sampling plan has an advantage in terms of shorter test time, a saving of resources, and in which a specific fraction of individuals at risk may be removed from the experiment at each of several ordered failure times. The familiar complete, type II right censored, first-failure-censored, and progressively type II right censored samples are special cases of the progressive first-failure-censored sampling plan. The Weibull distribution has been recognized as a useful model for the analysis of lifetime data. So, we consider the case of the progressive first-failure-censored sampling plan, and our study applied the large-sample theory to construct an MLE of $C_{L}$ under the Weibull distribution. Moreover, the MLE of $C_{L}$ is utilized to develop a new testing procedure for the performance index of products. The new hypothesis testing procedure 
is a quality performance assessment system in Enterprise Resource Planning (ERP). The managers can then employ the new testing procedure to determine whether the lifetime performance of products adheres to the required level. The managers can also utilize this procedure to enhance product process capability.

\section{Acknowledgments}

The authors wish to thank the referee for valuable suggestions which led to the improvement of this paper. This paper was partially supported by the National Science Council, Taiwan (Plan no. NSC 100-2221-E-158-004).

\section{References}

[1] D. C. Montgomery, Introduction to Statistical Quality Control, John Wiley \& Sons, New York, NY, USA, 1985.

[2] V. E. Kane, "Process capability indices," Journal of Quality Technology, vol. 18, pp. 41-52, 1986.

[3] L. I. Tong, K. S. Chen, and H. T. Chen, "Statistical testing for assessing the performance of lifetime index of electronic components with exponential distribution," International Journal of Quality $\mathcal{E}$ Reliability Management, vol. 19, pp. 812-824, 2002.

[4] C.-W. Hong, J.-W. Wu, and C.-H. Cheng, "Computational procedure of performance assessment of lifetime index of businesses for the Pareto lifetime model with the right type II censored sample," Applied Mathematics and Computation, vol. 184, no. 2, pp. 336-350, 2007.

[5] W.-C. Lee, J.-W. Wu, and C.-W. Hong, "Assessing the lifetime performance index of products from progressively type II right censored data using Burr XII model," Mathematics and Computers in Simulation, vol. 79, no. 7, pp. 2167-2179, 2009.

[6] W. C. Lee, J. W. Wu, C. W. Hong, H. Y. Pan, and W. L. Hung, "Decision procedure of performance assessment of lifetime index of products for the Gompertz distribution," Proceedings of the IMechE, Part B, vol. 224, no. 3, pp. 493-499, 2010.

[7] W. Weibull, "A statistical distribution function of wide 4pplicability," Journal of Applied Mechanics, vol. 18, pp. 293-297, 1951.

[8] W. Nelson, Applied life Data Analysis, John Wiley \& Sons, New York, NY, USA, 1982.

[9] L. G. Johnson, Theory and Technique of Variation Research, Elsevier, 1964.

[10] U. Balasooriya, "Failure-censored reliability sampling plans for the exponential distribution," Journal of Statistical Computation and Simulation, vol. 52, pp. 337-349, 1995.

[11] J. W. Wu, T. R. Tsai, and L. Y. Ouyang, "Limited failure-censored life test for the weibull distribution," IEEE Transactions on Reliability, vol. 53, pp. 107-111, 2001.

[12] S.-J. Wu and C. Kuş, "On estimation based on progressive first-failure-censored sampling," Computational Statistics \& Data Analysis, vol. 53, no. 10, pp. 3659-3670, 2009.

[13] A. J. Fernández, "On estimating exponential parameters with general type II progressive censoring," Journal of Statistical Planning and Inference, vol. 121, no. 1, pp. 135-147, 2004.

[14] P. K. Sen, "Progressive censoring schemes," in Encyclopedia of Statistical Sciences, S. Kotz and N. L. Johnson, Eds., vol. 7, pp. 296-299, John Wiley \& Sons, New York, NY, USA, 1986.

[15] P. W. Zehna, "Invariance of maximum likelihood estimators," Annals of Mathematical Statistics, vol. 37, no. 3, article 744, 1966.

[16] P. K. Sen and J. M. Singer, Large Sample Methods in Statistics, Chapman \& Hall, New York, NY, USA, 1993.

[17] G. Casella and R. L. Berger, Statistical Inference, Duxbury, Pacific Grove, Calif, USA, 2nd edition, 2002.

[18] J. F. Lawless, Statistical Models and Methods for Lifetime Data, Wiley Series in Probability and Statistics, Wiley-Interscience, Hoboken, NJ, USA, 2nd edition, 2003. 


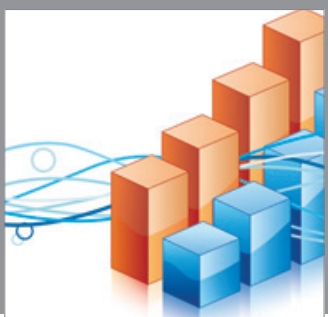

Advances in

Operations Research

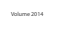

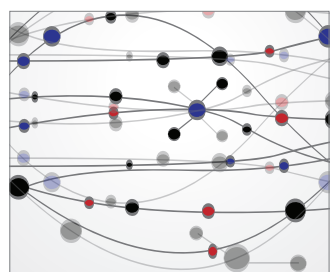

\section{The Scientific} World Journal
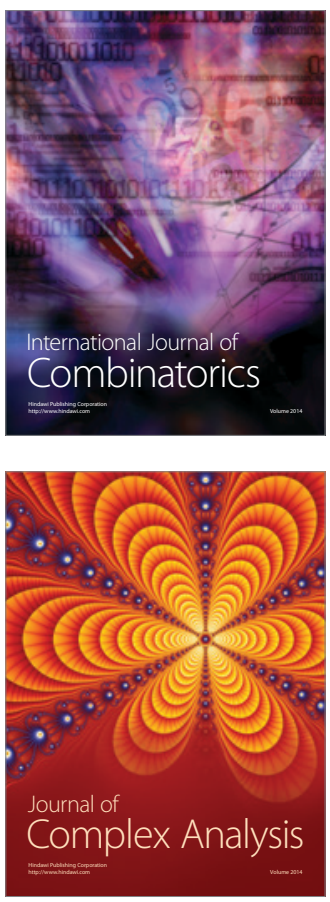

International Journal of

Mathematics and

Mathematical

Sciences
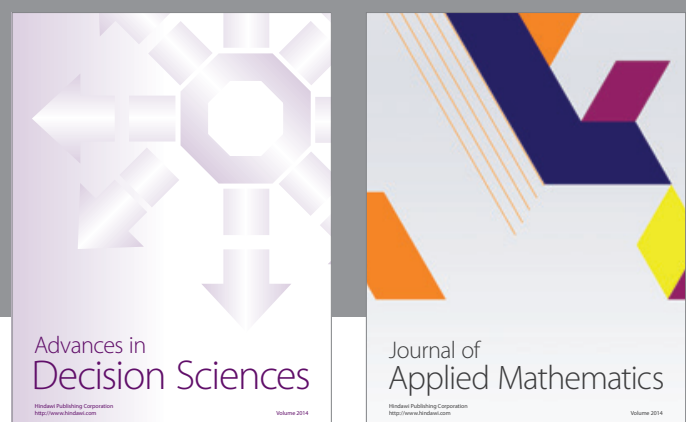

Journal of

Applied Mathematics
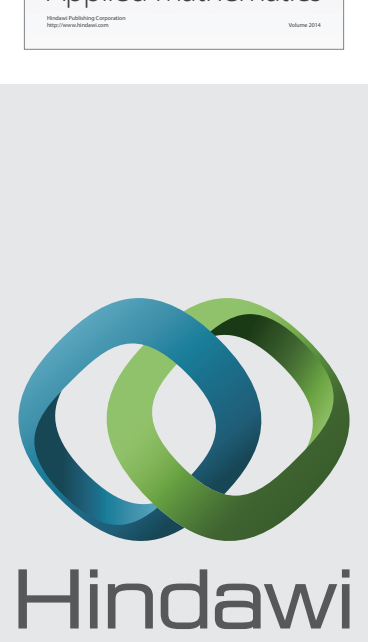

Submit your manuscripts at http://www.hindawi.com
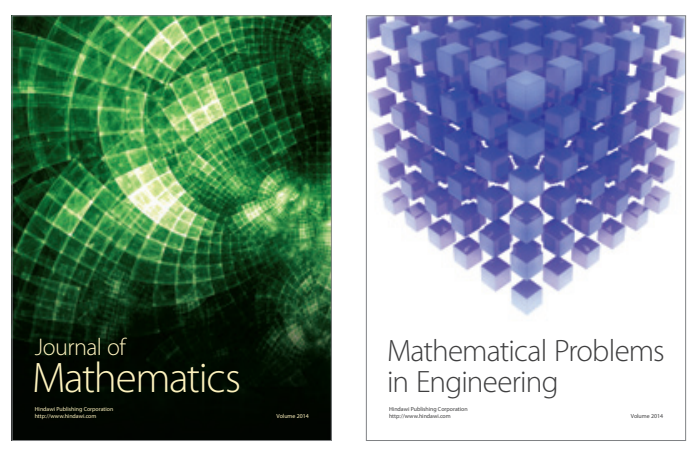

Mathematical Problems in Engineering
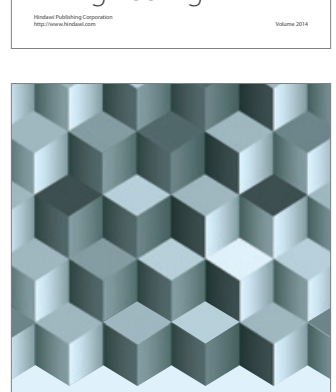

Journal of

Function Spaces
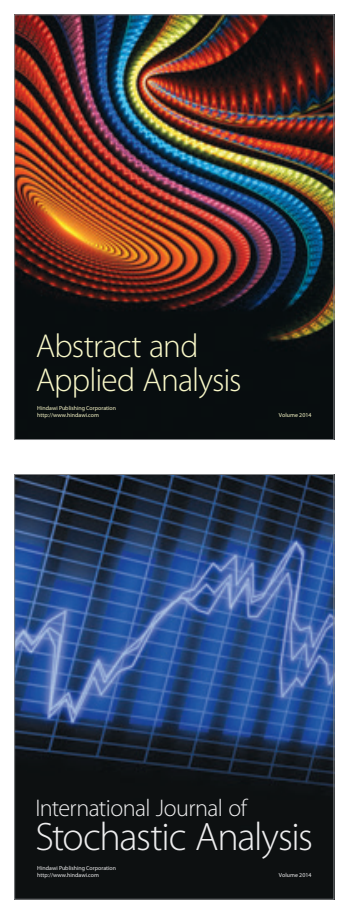

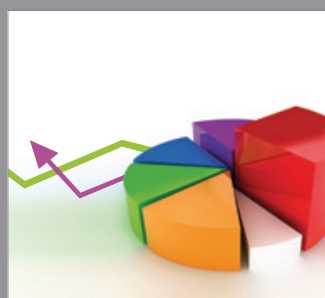

ournal of

Probability and Statistics

Promensencen
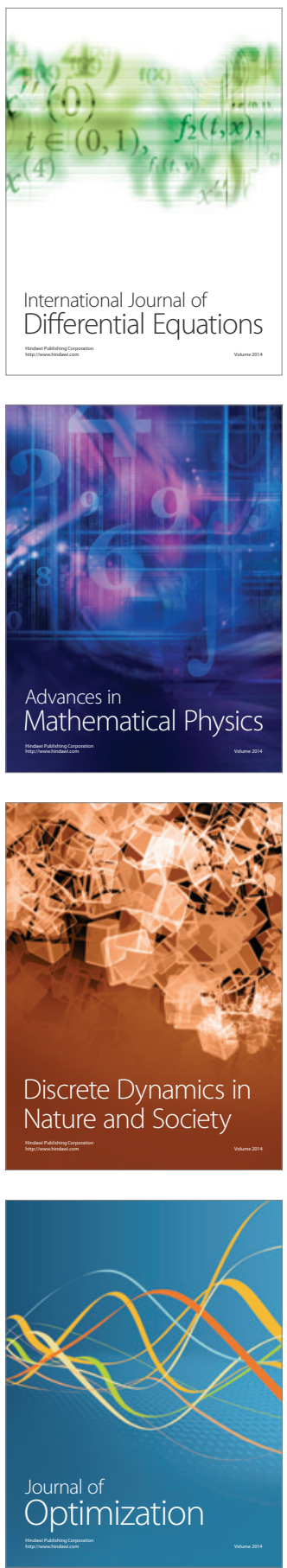